Wunderlich, $U$.

Suizidales Verhalten im Jugendalter.

Theorien, Erklärungsmodelle und Risikofaktoren

Göttingen, Hogrefe, 2004, 133 S., 24,95 EUR

ISBN 3-8017-1833-6

Die Suizidversuchsrate bei Jugendlichen und jungen Erwachsenen, insbesondere Frauen zwischen 15 und 24 Jahren ist alarmierend. Aus diesem Grund kommt der Erforschung von Risikofaktoren und präventiven Maßnahmen für suizidale Handlungen eine bedeutende Rolle zu. Dieses Buch erörtert umfassend, aber prägnant verschiedene Theorien und Modelle zu den Entstehungsbedingungen von Suizidalität sowie aktuelle Ergebnisse aus der Risikoforschung.

Zunächst werden Begriffsbestimmungen und Epidemiologie der Suizidalität beschrieben. Soziologische und psychologische, insbesondere psychoanalytische, psychodynamische und lerntheoretische Modelle werden leicht verständlich im Überblick vorgestellt. Dabei wird wichtige Literatur deutscher und internationaler Autoren berücksichtigt. Den Jugendlichentheorien wird ein eigenes Kapitel gewidmet, in dem das Konstrukt der Resilienz sowie der Vergleich von resilienten und suizidalen Jugendlichen erörtert werden. Des Weiteren werden wesentliche Befunde zu möglichen und gesicherten Risikofaktoren für Suizidalität zusammengefasst. Trotz der sehr trockenen Thematik ist der Autorin eine unkomplizierte und gut verständliche Darstellung gelungen. Wichtige Studien zur Untersuchung von Risikofaktoren für Suizid und Suizidversuche, insbesondere verschiedenen psychischen Störungen, werden erwähnt und knapp zusammengefasst. Biologische Erklärungsansätze werden ebenfalls diskutiert. Im nächsten Kapitel gibt die Autorin einen kurzen, aber prägnanten Überblick über multikonditionale Modelle zur Suizidalität, einschließlich des Overlap-Modells. Das umfangreiche Kapitel über die methodisch gut abgesicherte Dissertationsarbeit der Autorin ist besonders spannend. Es wird eine breit angelegte Verlaufsstudie mit einem Nachbeobachtungszeitraum von etwa 5 Jahren vorgestellt, in die 3021 Probanden aus einer repräsentativen Bevölkerungsstichprobe von 14- bis 24-jährigen Jugendlichen und jungen Erwachsenen einbezogen wurden. Ziel war die Untersuchung von Risikofaktoren für Suizidversuche. Die Darstellung der Ergebnisse und der Methodik ist exzellent. Folgende Risikofaktoren für Suizidversuche wurden in der Forschungsarbeit für ein erhöhtes Suizidversuchsrisiko als ausschlaggebend identifiziert und miteinander in Beziehung gesetzt: soziodemographische Variablen wie weibliches Geschlecht, psychische Störungen, insbesondere Angststörun- gen, Dysthymie und bipolare Störung, sowie Komorbidität mehrerer psychischer Störungen. Traumatische bzw. negative Lebensereignisse, vor allem sexueller Missbrauch und Vergewaltigung bei Mädchen und jungen Frauen, ungünstige familiäre Bedingungen und körperliche Erkrankungen wurden als Risikofaktoren für Suizidversuchsverhalten bei Jugendlichen und jungen Erwachsenen bestätigt. Ausführliche Einzelfallbeschreibungen erhöhen die Plastizität der Untersuchungsergebnisse.

Das vorliegende Buch liefert erstmalig im deutschen Sprachraum eine umfassende Darstellung der Risikofaktoren für Suizidversuche im Jugendalter und schließt damit eine Forschungslücke. Es trägt zu einem tieferen und differenzierteren Verständnis multifaktorieller Zusammenhänge von Suizidversuchen im Jugendalter bei und liefert Anregungen für präventive Maßnahmen. Ein in Form und Inhalt ansprechendes und gelungenes Buch!

Barbara Schneider, Frankfurt/M.

Ketelsen, R.; Schulz, M.; Zechert, C.

Seelische Krise und Aggressivität.

Der Umgang mit Deeskalation und Zwang

Bonn, Psychiatrie-Verlag, 2004, 191 S., 19,90 EUR

ISBN 3-88414-376-X

Dieses Buch ist ein interessanter und wichtiger Beitrag zu einer noch immer tabuisierten Dimension der stationären Psychiatrie. Aus wenigen lokalen Untersuchungen weiß man, dass bei $5-10 \%$ der stationären Patienten Zwangsmaßnahmen wie Fixierung, Isolierung, Zwangsmedikation, Unterbringung gegen den Willen der Patienten eingesetzt werden. Die Autoren haben sich dieses Themas angenommen. Sie gehen den Fragen nach der Verantwortung, der Macht der in der Psychiatrie Tätigen, dem Einsatz von Zwang im Notfall und der Indikation für Zwangsmaßnahmen nach.

In der Einführung wird die unsystematische Vermittlung von Wissen und Techniken in der Aus- und Weiterbildung beklagt. Die Forschungslage zu Zwangsmaßnahmen («dirty work») in der stationären Psychiatrie ist uneinheitlich. Auffällig ist der regionale Unterschied in der Häufigkeit von Zwangsmaßnahmen. Im Kapitel «Grundlagen» wird aufbauend auf die Begriffsklärung von Zwang, Gewalt, Aggression und Macht ein generelles Präventionsmodell vorgestellt. Als primäre Prävention werden die vorbeugende Arbeit und das Erkennen von Risikofaktoren, als sekundäre Prävention das Vorgehen 
bei akutem auto- und fremdaggressivem Verhalten und als tertiäre Prävention die Nachsorge nach aggressiven Vorfällen verstanden. Die Darstellung der Stufen der Eskalation und daraus abgeleitete Maßnahmen und Behandlungshinweise werden von Praktikern als hilfreich eingeschätzt. Die Autoren arbeiten einen Katalog von Indikationskriterien für Zwangsmaßnahmen heraus, wobei sie feststellen, dass die Indikationen eher auf Tradition als auf Studien beruhen. Im Kapitel «Notfall» werden ausführlich die Aspekte der sekundären Prävention behandelt: Ort des Geschehens, Phasenmodell aggressiver Vorfälle (Auslösephase, Eskalationsphase, Krise, Erholungsphase, Depression nach der Krise). Für die tertiäre Prävention werden Standards für Nachbesprechungen und zur Bearbeitung des Erlebens von Zwangsmaßnahmen bei Patienten und Mitarbeitern dargestellt. Die Möglichkeit einer Retraumatisierung gerade auf Patientenseite wird angesprochen. In den «speziellen Grundlagen» wird die medikamentöse Behandlung des psychiatrischen Notfalls dargestellt. Die Ausführungen zu Zwangsmaßnahmen in der Gerontopsychiatrie weisen auf die dortige Brisanz hin, da beispielsweise Bettgitter und Stuhlbretter auch außerhalb der Psychiatrie in der Altenarbeit häufig vorkommend. Dazu gehören auch subtile Gewaltformen wie Wartenlassen, Unterlassungen von Kleidungswechsel und manche Art der Nahrungsdarreichung. Im Kapitel «Qualitätssicherung und Weiterbildung» wird die Vernachlässigung der Zwangsmaßnahmen im Rahmen der allerorts betriebenen Qualitätssicherungen beklagt, ebenso die wenig aussagekräftigen Vergleichsstudien. Als Weg für die notwendige Verbesserung werden die Einrichtung von Qualitätszirkeln in psychiatrischen Einrichtungen und die Einführung von Standards für die Durchführung von Zwangsmaßnahmen vorgeschlagen. Die vorgestellten «10 Schritte zur Verbesserung» und Qualitätssicherung bei Zwangsmaßnahmen sollten künftig selbstverständlicher Standard und Teil von Leitlinien sein. In Kliniken zu organisierende Beraterteams müssen Trainingsmaßnahmen zur Gewaltprävention (Stressmanagement, adäquate Situationseinschätzung, aktive Deeskalation und Anwendung schonender Abwehrtechniken) erfahren. Wesentliches Ziel ist die Beherrschung der Situationen, nicht der Patienten.

Fazit: Professioneller Umgang mit Zwangsmaßnahmen setzt präventive Strategien, trainierte Mitarbeiter, Enttabuisierung, ein effektives Stationsmilieu und mehr Studien über die Entstehung autoaggressiven Verhaltens voraus. Die im Anhang dargestellten «Bielefelder Standards» können Grundlage für einen Konsens für die Professionellen darstellen. - Ein wichtiges Buch, dem wir eine Verbreitung in allen psychiatrischen Institutionen wünschen. Es sollte Pflichtlektüre in der Weiterund Fachausbildung der psychiatrischen Profis sein.

Siegfried Traxler, Andernach

\section{Trautmann, R.D.}

\section{Verhaltenstherapie bei Persönlichkeitsstörungen und problematischen Persönlichkeitsstilen \\ Reihe: Leben lernen, Bd. 176}

Stuttgart, Pfeiffer bei Klett-Cotta, 179 S., 20,- EUR

ISBN 3-608-89721-6

Die psychotherapeutische Behandlung von Persönlichkeitsstörungen stellt eine der größten Herausforderungen der klinischen Praxis dar. Das vorliegende Buch richtet sich weniger an Berufseinsteiger, als vielmehr an Personen, die schon Erfahrung mit der Behandlung von Patienten mit Persönlichkeitsstörungen haben. Bereits im Vorwort weist der Autor darauf hin, dass sein Anliegen vor allem darin liege, «aus der Praxis heraus anderen Praktikern konkrete Hilfen an die Hand zu geben ...».

Das Buch ist gegliedert in sieben aufeinander aufbauende Kapitel. Im ersten Abschnitt des Buches (Kap. 1 und 2) wird ausführlich auf den derzeitigen Stand sowie die Schwierigkeiten bei der Diagnostik und Klassifikation von Persönlichkeitsstörungen eingegangen. Besonders berücksichtigt wird dabei die Unterscheidung zwischen Persönlichkeitsstilen und Persönlichkeitsstörungen sowie deren Implikationen für eine mögliche Behandlung. Unterschiedliche Definitionsmöglichkeiten der Begriffe Persönlichkeit, Persönlichkeitsstörung und Persönlichkeitsstil werden diskutiert. Besonderer Raum wird dabei den theoretischen Ansätzen von Millon [1996] eingeräumt, auf die sich der Autor in den weiteren Kapiteln des Buches immer wieder bezieht. Allgemeine Grundprinzipien verhaltenstherapeutischen Vorgehens wie Transparenz oder Zielorientierung hat der zweite Abschnitt des Buches zum Inhalt. Hier behandelt der Autor zunächst die allgemeine Auslegung einzelner Grundprinzipien. Anschließend richtet er sein Augenmerk auf die Anforderungen dieser Grundprinzipien in der Therapie verschiedener Persönlichkeitsstörungen. Menschen mit Persönlichkeitsstörungen erleben Emotionen völlig anders. Insofern scheint es folgerichtig, dass auch im vorliegenden Werk ein Kapitel mit der Überschrift «Die Emotionen bei Persönlichkeitsstörungen» folgt. Hier wird im Besonderen auf die andere, existentiell erlebte Qualität von Emotionen hingewiesen, die es aus Sicht des Autors bei der Therapie besonders zu berücksichtigen und stets zu vergegenwärtigen gilt. Als zentralen Punkt im Buch führt der Autor im 6. Kapitel geeignete Vorgehensweisen bei den einzelnen Persönlichkeitsstörungen jeweils kurz, aber prägnant aus. $\mathrm{Zu}$ jedem einzelnen Störungsbild werden eigene theoretische Überlegungen, Besonderheiten der Diagnostik sowie wichtige Aspekte der Therapie und Beziehungsgestaltung erklärt, vorgestellt und ausgeführt. Dabei stehen aus der praktischen Erfahrung des Autors entstandene Erklärungsmodelle im Vordergrund. Sie sollen es Patienten ermöglichen, Zugang zur Entstehung der eigenen Problematik zu bekommen und plausible und annehmbare Therapieziele zu entwickeln. Durch leicht verständliche und konkrete Fallbeispiele aus der Praxis 
werden diese Vorgehensweisen veranschaulicht und untermauert. Besonders wird die Unterscheidung von Psychotherapie bei und Psychotherapie der Persönlichkeitsstörung als zentral für die Konzeption und Umsetzung therapeutischer Vorgehensweisen betont. Hier weist der Autor explizit auf den Unterschied der verschiedenen Behandlungsvarianten unter Berücksichtigung der jeweiligen Motivation des Patienten hin. Die Zielsetzung sollte sich hierbei zusätzlich sowohl nach den äußeren Rahmenbedingungen als auch nach Voraussetzungen auf Seiten des Patienten wie dessen Alter oder den Ausprägungsgrad der Störung richten.

Insgesamt erhält der interessierte Leser umfassenden Einblick in die Besonderheiten im therapeutischen Umgang mit Patienten mit Persönlichkeitsstörungen und problematischen Persönlichkeitsstilen. Durch die eingestreuten Fallbeispiele werden dem Leser eindrücklich und für den Fachmann leicht verständlich die Ansätze des Autors nahe gebracht. Dem Anspruch, ein Buch für Fortgeschrittene zu verfassen, wurde der Autor gerecht. Das Buch kann dem Therapeuten Hilfestellung geben, leichter eigene Ideen und Strategien bei der Behandlung persönlichkeitsgestörter Menschen zu entwickeln.

Sabine Kaiser, Mainz

Millon T: Disorders of Personality. DSM IV and Beyond, ed 2. New York, Wiley, 1996.

Wewetzer, C. (Hrsg)

\section{Zwänge bei Kindern und Jugendlichen}

Göttingen, Hogrefe, 2004, 144 S., 24,95 EUR

ISBN 3-8017-1739-9

Zwangsgedanken und Zwangshandlungen sind für betroffene Kinder und Jugendliche sehr belastend. Noch weniger als betroffene Erwachsene können sie diese einordnen, sich dazu äußern oder sich um Hilfe bemühen. Dadurch ist auch das Familiensystem auf eine harte Probe gestellt, aus Unwissen kommt es zu falschen Zuschreibungen, das Problem wird meist sehr spät erkannt. Der professionelle Helfer ist an dieser Stelle gefragt und oftmals vor keine leichte Aufgabe gestellt. Umso nützlicher ist ein Buch, das sich ausschließlich diesem Störungsbild im Kindes- und Jugendalter widmet. Ziel des Buches ist, so Wewetzer und Warnke in der Einleitung, die Vermittlung von aktuellem Wissen sowie Kenntnissen zum Verständnis des Krankheitsbildes. Zudem nennen sie ihr Anliegen, dem Kliniker Hilfen für die therapeutische Arbeit zu geben. Bei der Betrachtung des Inhaltsverzeichnisses wird ersichtlich, dass die Autoren den Schwerpunkt auf die Darstellung neuester Studien und Erkenntnisse zur Ätiologie und Genese sowie zum Verlauf von Zwangsstörungen gelegt haben. Denn die Kapitel, die sich auf die Therapie der Zwangsstörung im Kindes- und Jugendalter beziehen, bleiben im Vergleich überschaubar. Das Buch ist in acht Kapitel untergliedert. In den ersten beiden Kapiteln (Einleitung und Epidemiologie) wird ein kurzer historischer Überblick gegeben, anschließend folgen Erläuterungen zu Definition, Klassifikation und Diagnostik sowie Differentialdiagnostik. Es schließt ein kurzer Überblick zu den Prävalenzen, Inzidenzen, Erkrankungsalter und der Geschlechterverteilung an. In Abgrenzung zu Zwangshandlungen wird zudem auf die entwicklungstypischen Rituale bei Kindern und Jugendlichen eingegangen - ein sehr wichtiger Aspekt. Den beiden ersten, kurz gehaltenen Kapiteln folgt ein ausführlicher Überblick über die Symptomatik und die komorbiden Störungen (Achse I und II). Hatte der Leser sich auf eine ausführliche Darstellung der Symptomatik der juvenilen Zwangsstörung auch in Abgrenzung zur Zwangsstörung bei Erwachsenen gefreut, wird er enttäuscht, denn es wird sehr viel ausführlicher auf die komorbiden Störungen eingegangen. In dem 4. und längsten Kapitel des Buches fassen die Autoren den gegenwärtigen Kenntnisstand einer multifaktoriellen Genese der Zwangsstörung zusammen. Sie gehen dabei unter anderem auf den psychoanalytischen und lerntheoretischen Erklärungsansatz ein. Sehr ausführlich werden auch der neuroanatomische Erklärungsansatz sowie Ergebnisse zu genetischen Untersuchungen vorgestellt. Kapitel 5 wird den familiären und psychosozialen Aspekten bei der Entwicklung der Zwangsstörung gewidmet. Hier werden in einem gelungenen Überblick familiäre Faktoren wie z.B. hohe Leistungsorientierung oder «high-expressed emotions» (HEE) und ihr Einfluss auf die Begünstigung bzw. Aufrechterhaltung einer Zwangsstörung diskutiert. In Kapitel 6 folgt eine Darstellung verhaltenstherapeutischer Interventionen. Im Vergleich zu den ausführlich dargestellten Erkenntnisse zur Ätiologie und Genese der Störung in den vorangegangenen Kapiteln bleibt das Kapitel zu den verhaltenstherapeutischen Interventionen recht knapp. Hier wäre eine vertiefte Darstellung des therapeutischen Vorgehens z.B. mit eingehender Beschreibung des «Home-treatment-Ansatzes» sehr hilfreich gewesen. Das Buch schließt mit der Pharmakotherapie (Kap. 7) sowie dem Verlauf von Zwangsstörungen (Kap. 8).

Abschließend bleibt zu bemerken, dass für den Kliniker, der mit dem Kind/Jugendlichen und dessen Familie die Zwangsstörung behandelt, die Ausführungen über die therapeutische Arbeit zu kurz sind, um sie tatsächlich in der Praxis anwenden zu können. Insgesamt ist dieses Buch jedoch für den Einstieg in das Thema sicherlich sehr empfehlenswert. Auch für den interessierten Leser, der sich in die neuesten Erkenntnisse zu den Wechselwirkungen zwischen biologischen und psychosozialen Entwicklungsdeterminanten einlesen möchte, ist es wertvoll.

Heike Lau, Wiesbaden 
Bischoff, C.; Traue, H.C.

Kopfschmerzen

Reihe: Fortschritte der Psychotherapie -

Manuale für die Praxis, Band 22

Göttingen, Hogrefe, 2004, 116 S., 19,95 EUR

ISBN 3-8017-1623-6

«Kopfschmerzen zählen zu den häufigsten Erkrankungen.» So beginnt der Text auf der Umschlagseite des 22. Bandes der Reihe Fortschritte der Psychotherapie - Manuale für die Praxis. Claus Bischoff und Harald C. Traue widmen sich darin dieser häufigen Erkrankung und vermitteln dem Verhaltensmediziner in komprimierter Form grundlegendes Störungsund Behandlungswissen. Dass dieses gefragt ist, verdeutlicht nicht nur die Häufigkeit von Kopfschmerzerkrankungen, sondern offenbar auch die Nachfrage nach Behandlern mit entsprechendem Expertenwissen. So beschreibt auch May im Editorial der letzten Ausgabe der Zeitschrift «Der Schmerz», dass sich der Löwenanteil der Patientenanfragen an eine der wichtigsten deutschen Fachgesellschaften für den Bereich Kopfschmerzen, die Deutsche Migräne- und Kopfschmerzgesellschaft (DMGK), nicht auf Diagnostik und Therapie, sondern schlicht auf die Frage nach Kopfschmerzexperten in räumlicher Nähe beziehe.

Im ersten Kapitel des Bandes beschreiben die Autoren die für den Verhaltensmediziner bedeutsamsten Kopfschmerzformen Migräne, Kopfschmerz vom Spannungstyp und Kopfschmerz, der mit übermäßiger Einnahme von Schmerzmitteln oder deren Entzug verknüpft ist, und stellen deren Verankerung in den Klassifikationssystemen der IHS und dem ICD dar. Kurzes Basiswissen über den Clusterkopfschmerz, den zervikogenen Kopfschmerz und chronischen posttraumatischen Kopfschmerz ist dem Kapitel beigefügt. Als sehr hilfreich für den praktisch arbeitenden Verhaltensmediziner sind in diesem Kapitel auch die Hinweise zur Differential- und Komorbiditätsdiagnostik. Das 2. Kapitel gibt einen Überblick über Störungstheorien und Modelle zum Kopfschmerz. Zu den beschriebenen Kopfschmerzformen wird jeweils der aktuelle Wissensstand $\mathrm{zu}$ Pathopsychologie, Pathophysiologie und Auslösern kurz dargestellt. Positiv hervorzuheben ist dabei, dass auch viele neuere Forschungsergebnisse Eingang in diese
Kapitel gefunden haben. Auf drei Karten in der Umschlaginnenseite sind die Informationen dann zusätzlich noch in einem jeweiligen Erklärungsmodell in plausibler und den Patienten vermittelbarer Form skizziert. Umfassend ist die Darstellung der diagnostischen Verfahren und Dokumentationshilfen im 3. Kapitel. Besonders das Vorgehen bei Erhebung der Schmerzanamnese wird von den Autoren sehr übersichtlich aufgebaut. Die Darstellung der verhaltensmedizinischen Interventionen beschränkt sich auf die Behandlung von Migräne, Kopfschmerzen vom Spannungstyp und medikamenteninduzierten Kopfschmerzen. Vorangestellt werden Informationen zur Indikation und Kontraindikation sowie Hinweise darauf, welche Behandlungsmaßnahmen im Sinne einer Prioritätenliste bei diesen Kopfschmerzformen Vorrang haben sollten. Die einzelnen Behandlungsmaßnahmen werden anschließend jedoch nur kurz beschrieben. Als ein manualgesteuertes multimodales verhaltensmedizinisches Programm zur Kopfschmerztherapie stellen die Autoren zusätzlich die Konkordanztherapie in groben Zügen vor. Ein kleiner Exkurs mit Hinweisen zur Diagnostik und Behandlung von Kopfschmerz bei Kindern folgt. «Psychotherapie bei Kopfschmerz ist evidenzbasiert.» Diese Aussage im letzten Kapitel wird durch die abschließende gute Zusammenstellung der empirischen Evidenzen unterstrichen.

Wie an der Auflistung der Inhalte deutlich wird, haben die Autoren viele Informationen zum Kopfschmerz zusammengestellt. Manches ist dabei jedoch sehr kompakt und leider auch etwas knapp geraten. Vielleicht hätten der Verzicht auf einzelne Themen wie Diagnostik und Kopfschmerz bei Kindern oder Darstellung einer multimodalen Behandlungsform, zugunsten von mehr konkreten Informationen wie Beispielen bei den Behandlungsmaßnahmen die Lektüre noch hilfreicher und interessanter gemacht. Traue und Bischoff geben mit dem vorliegenden Band dennoch einen guten aktuellen Überblick zum Kopfschmerz, und damit dem Psychotherapeuten, der auch Patienten mit Kopfschmerzen behandelt, hilfreiches Hintergrundwissen. Zum Kopfschmerzexperten wird man mit der Lektüre des Buches hingegen nicht. Das kann ein knapp 120 Seiten umfassendes Buch aber auch nicht leisten.

Katrin Mauer-Matzen, Main 